Revista Digital Universitaria

Vol. 22, Núm. 6, noviembre-diciembre 2021

\title{
La importancia de la inteligencia emocional en la población masculina
}

Liliana Peralta Tellez

\begin{abstract}
Resumen
La inteligencia emocional es un término que se emplea para describir las cualidades emocionales para lograr el éxito. Existe una gran curiosidad por identificar los beneficios de la inteligencia emocional en la población masculina, para contrarrestar la violencia de género, delincuencia organizada, suicidios, etcétera, así como romper el sistema patriarcal que ha permanecido y ha sido socialmente aceptado en la población a pesar de los avances y retrocesos en la igualdad de género. Este artículo persigue dos objetivos: el primero es revisar los vínculos entre el tema de la inteligencia emocional en la población masculina y la violencia de género. El segundo es proponer la producción de nuevas líneas y áreas de investigación, para ofrecer puntos de reflexión, nuevos conocimientos, y generar así avances en el campo, al promover la inteligencia emocional en la población masculina.
\end{abstract}

Palabras clave: inteligencia emocional, población masculina, violencia de género.

\section{THE IMPORTANCE OF EMOTIONAL INTELLIGENCE IN THE MALE POPULATION}

\begin{abstract}
Emotional intelligence is a term that is used to describe the emotional qualities to achieve success. There is great interest to identify the benefits of emotional intelligence in the male population to counteract gender violence, organized crime, suicides, etc., as well as breaking the patriarchal system that has remained and has been socially accepted in the population despite advances and setbacks in gender equality. This article has two objectives. The first one is to review the links between emotional intelligence in the male population and gender violence. The second one is to propoese the production of new lines and areas of research in the subject, offering points of reflection and new knowledge in order to promote emotional intelligence in the male population.
\end{abstract}

Keywords: emotional intelligence, male population, gender violence. 


\section{Liliana Peralta Tellez}

Obtuvo el título de Técnico en Informática por el Colegio de Estudios Científicos y Tecnológicos del Estado de México, plantel Coatepec Harinas; el título de Licenciada en Educación Primaria por la Escuela Normal de Coatepec Harinas con la tesis "Cómo favorecer la formación ciudadana a partir de la promoción y práctica de valores en el segundo grado grupo 'B' de la escuela primaria Lic. Benito Juárez", y el título de Maestra en Educación Basada en Competencias por la Universidad del Valle de México, Campus Chapultepec. Acreditó el curso "Gestion estratégica de instituciones educativas" por la Facultad de Educación y Ciencias Sociales de la Universidad Andrés Bello, y el curso "Cuidando la salud mental de las niñas, niños y adolescentes en el regreso a la escuela. Estrategias de intervención para profesores en el salón de clases", impartido por Dirección General de Gestión Escolar y Enfoque Territorial de la sEB. Es profesora de educación primaria en la escuela Guadalupe Victoria con сст 15EPR0122I, en Coatepec Harinas, Estado de México. Se ha desempeñado como profesora titular de asignaturas como estrategias de aprendizaje, metodología de la investigación, diseño curricular, teorías del aprendizaje, modelos y técnicas de enseñanza, liderazgo y calidad educativa, administración educativa, entre otras, en la Universidad "Grupo Educativo Uninet" con сcт 15PCT0107G campus Sultepec, Estado de México y campus Coatepec Harinas, Estado de México. 


\section{Introducción: situación actual en México}

Te has puesto a pensar qué hay detrás de las cifras de feminicidios? Y es que estos homicidios cometidos contra mujeres por razones de género van en aumento: "el 2020 fue el año con más feminicidios en México, alcanzando 969 víctimas" (Causa en Común, 2021). Vale la pena destacar que en ellos, la mayoría de los victimarios mantenían una relación familiar cercana a la víctima.

La violencia familiar también alcanzó un "nivel máximo histórico. De 210,158 denuncias registradas en 2019, se pasó a 220,028 en 2020, lo que representa un aumento del 5\%" (Causa en Común, 2021). En el mismo sentido, las estadísticas de suicidios donde "los hombres tienen una tasa de 8.9 fallecimientos por cada 100 mil hombres (5 454), mientras que esta situación se da en 2 de cada 100 mil mujeres (1 253)" (INEGI, 2020, p.1).

Asimismo, ha habido un incremento de la delincuencia organizada, que de acuerdo a las cifras oficiales "ha aumentado en un $40.5 \%$ desde 2015 " (Índice de Paz México, 2021). En este rubro "noventa por ciento de todas las víctimas de homicidio son hombres, y la mayoría de estos homicidios están vinculados a dinámicas de la delincuencia organizada" (Índice de Paz México, 2021). De acuerdo con el informe del Índice de Paz en México (2021), el "homicidio fue la principal causa de muerte entre los hombres de 10 a 54 años, por el contrario, es más probable que las muertes de mujeres estén asociadas a la violencia de pareja".

De esta manera, vale la pena destacar que en nuestra cultura, en general, se ha enfocado en establecer la figura de los hombres como líderes, proveedores, fuertes, los que no lloran, los que no sufren, los exitosos, los que resuelven problemas, y muy poco se ha puesto la atención a su inteligencia emocional. Ciertamente, en la actualidad luchamos por la equidad e igualdad de género, y en esta lucha también es importante voltear a ver a esta población masculina. Ahí radica la importancia de este artículo, en despertar el interés del lector por seguir investigando sobre este tema: la inteligencia emocional en los hombres y el impacto que puede tener en temas tan complejos como los ya mencionados.

\section{¿Qué es la inteligencia emocional?}

La primera vez que se utilizó el término inteligencia emocional fue en 1990, por los psicólogos Peter Salovey y John D. Mayer, como las cualidades emocionales necesariar para lograr el éxito (Baena, 2003, p. 32). En este artículo se define al éxito como la capacidad de mantener un estado de bienestar y plenitud personal, que lleva al ser a sentirse feliz por lo que ha logrado consigo mismo. Es capaz de desarrollarse en ambientes sanos, productivos, y puede establecer relaciones sociales asertivas y, por ende, no afecta a otras personas. 


\section{IMPORTANCIA DE} LA INTELIGENCIA EMOCIONAL (IE)

cualidades emocionales para

lograr el éxito

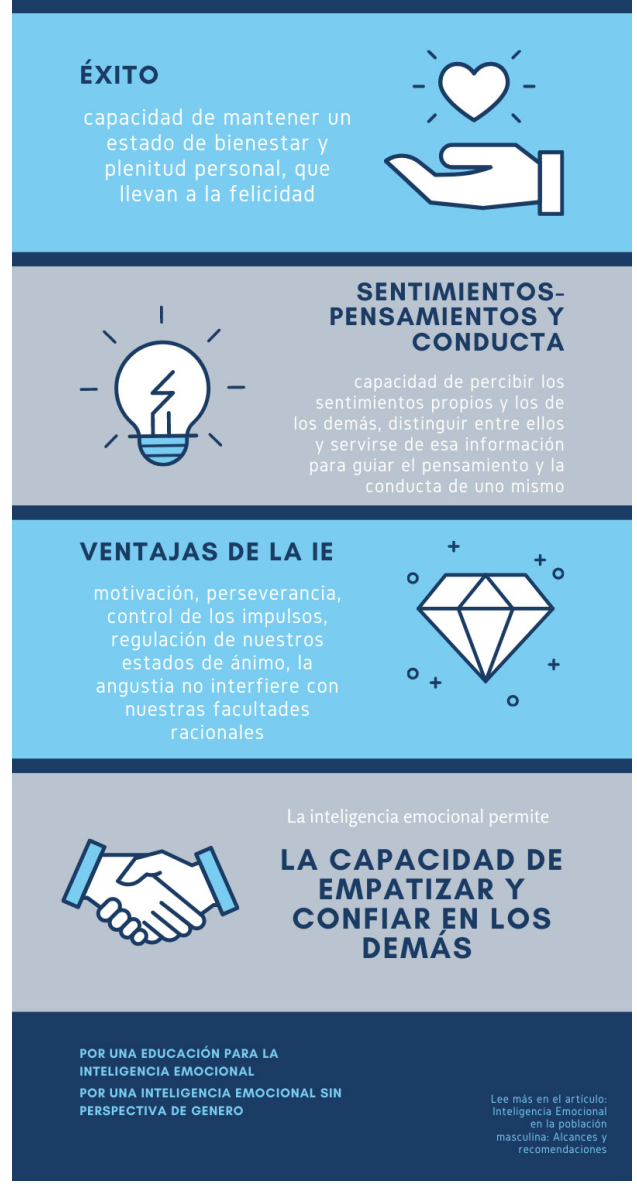

En este contexto, tiene sentido la definición que Grewal y Salovey proporcionaron de la inteligencia emocional, pues para ellos es "la capacidad de percibir los sentimientos propios y los de los demás, distinguir entre ellos y servirse de esa información para guiar el pensamiento y la conducta de uno mismo" (2006, p.13).

Así, es interesante reflexionar sobre qué tan hábiles somos en reconocer lo que sentimos y siente otra persona, cómo gestionamos esos sentimientos para actuar de una manera determinada, sin dañar a la otra parte ni a uno mismo. De esta manera, cuando una persona logra equilibrar su inteligencia emocional, puede mantener relaciones sociales saludables.

Por ello, es necesario voltear a ver a los hombres que han participado en actos de violencia, que han pensado en el suicidio, que se han incorporado a las líneas de grupos de la delincuencia organizada, a niños y jóvenes que han vivido separación, divorcio o abandono de sus padres, jóvenes y/o adultos que han abandonado a sus familias, etcétera y averiguar qué pasa desde la inteligencia emocional. Asimismo, hay que reflexionar cómo podemos cambiar la perspectiva de la educación a partir de los primeros años, para promover la inteligencia emocional desde la infancia. Goleman:

Entre las características de la inteligencia emocional destacan, según

La capacidad de motivarnos a nosotros mismos, de perseverar en el empeño a pesar de las posibles frustraciones, de controlar los impulsos, de diferir las gratificaciones, de regular nuestros propios estados de ánimo, de evitar que la angustia interfiera con nuestras facultades racionales y, por último, pero no menos importante, la capacidad de empatizar y confiar en los demás (1995, p. 43).

Desde la revisión teórica del término inteligencia emocional, de sus implicaciones y características, se pretende en este texto analizar posibles 
"La importancia de la inteligencia emocional en la población masculina"

Liliana Peralta Tellez

Vol. 22, Núm. 6, noviembre-diciembre 2021

Revista Digital Universitaria

líneas de investigación enfocadas en la población masculina en relación con la inteligencia emocional y su impacto en la disminución de violencia de género, feminicidios, participación con grupos de la delincuencia organizada, suicidio y aumento de relaciones familiares sólidas y significativas (es decir, que los miembros de la familia se desarrollen en ambientes libres de violencia y con amplitud para expresar sus emociones y sentimientos sin importar el género).

\section{Inteligencia emocional:implicaciones en la población masculina}

En varios estudios se ha encontrado que:

los hombres tienden con mayor frecuencia a internalizar las emociones, es decir a mostrar una reacción fisiológica "emocional" ante un evento (es decir, su cuerpo reacciona ante la situación sudando, cambiando de color, principalmente enrojeciendo las mejillas, temblando, incluso sintiendo algún síntoma relacionado con alguna enfermedad), pero sin mostrar expresión emocional abierta de ello, por el contrario, las mujeres son más externalizadoras, es decir que se expresan en todos los planos (Brody, 2000; Vergara y Páez, 1989; Cova, 2004, citados en Ortega et al., 2013, p. 340).

Lo anterior permite reconocer que "existe una emocionalidad masculina inhibida o restrictiva, ya que la cultura determina cierta imposibilidad de expresión de algunas emociones" (Janz, 2000; Cova, 2004, citados en Ortega et al., 2013, p. 340). O sea, la cultura, la sociedad y las instituciones, incluida la familia, han fortalecido a través del tiempo las restricciones o impedimentos para que los niños, jóvenes y adultos puedan actuar y/o desempeñarse libremente tal cual son, demostrando sus emociones y sentimientos, exponiéndose frágiles y como individuos que también necesitan ayuda. Al contrario, se siguen reforzando ideologías machistas donde se cree que los hombres no lloran, no sufren, no tienen miedo, son valientes, no se quejan, no juegan con muñecas, lo que tiene un papel fundamental en la emocionalidad masculina inhibida.

Una de las mayores implicaciones de la inteligencia emocional en los hombres es, por tanto, su educación emocional desde la infancia en la familia, la cultura, las instituciones y la sociedad. Es necesario romper con los esquemas y con ello lograr líneas de investigación que den pautas desde el ámbito educativo para lograr una inteligencia emocional.

La investigación realizada por Ramírez relaciona la masculinidad con las emociones: "el poder como rasgo de la masculinidad cultural y patriarcal' se ha establecido desde las antiguas civilizaciones, aún permanece, por tanto, a los hombres no se les permite manifestarse como seres emocionales porque tienen el riesgo de perder el poder" $(2013$, p. 3).

Desde esta filosofía, "los hombres tienen menores niveles de inteligencia emocional por la conciencia contradictoria de lo que la sociedad espera de ellos", (Kaufman, 1997, p. 72, citado en Ramírez, 2013, p. 3). Por lo tanto, los hombres 
"La importancia de la inteligencia emocional en la población masculina"

Liliana Peralta Tellez

Vol. 22, Núm. 6, noviembre-diciembre 2021

Revista Digital Universitaria

deciden inhibir sus propias emociones con la idealización de continuar con el poder: si son expresivos emocionalmente creen que el poder que tienen sobre su grupo será derrotado y esto genera dolor para ellos, dolor que pueden expresar a través de actos de violencia o aislamiento, además de una emocionalidad inestable para cuidar y relacionarse con otros seres humanos. Con ello, "aumenta la solitaria búsqueda del poder y enfatiza nuestra convicción de que el poder requiere la capacidad de ser distante" (Kaufman, 1997, citado en Ramírez, 2013, p. 3).

La configuración de una práctica de género de un hombre opta por formas de control y dominación de sí mismo para poder hacerlo con los demás. Ello implica negar la expresión de emociones y de necesidades diversas (autocuidado, compasión, perdón. etc.), conlleva sufrimiento que no es posible manifestarlo porque sería signo de debilidad, feminización, generando temor, que se expresa como homofobia. Para afirmarse masculino tiene que mostrar prácticas acordes a la concepción dominante de masculinidad. Existe una relación directa entre temor-dolor-control. El dolor y las heridas son producto de la forma en que se ejerce el poder patriarcal (Ramírez, 2013, p.3).

Tanto hombres como mujeres se van construyendo como sujetos de género durante el ciclo vital. Desde la inteligencia emocional de los hombres en función de los estudios analizados, es importante considerar la categoría de la masculinidad que se les ha exigido a lo largo de la historia y cómo es que se puede romper ese esquema patriarcal, pues, además de que impide avanzar hacia la igualdad de género, genera temor, dolor, control en los hombres y con ello restricciones para que se demuestren como seres emocionales, que, al igual que las mujeres, necesitan reconocer sus emociones y sentimientos, valorarlos, trabajar en ellos y lograr una vida plena, para con ello mejorar su entorno social y cultural.

Es importante considerar que es necesario llevar a cabo un análisis profundo en futuras líneas de investigación sobre los sujetos de género, estigmas socioculturales y la mejora de los niveles de inteligencia emocional, para brindar bases en el sector masculino de la población y mejorar su desarrollo personal, la relación con sí mismos y con ello permitirles lograr las cualidades emocionales necesarias para alcanzar el éxito.

\section{Conclusiones}

La inteligencia emocional se entiende como las cualidades emocionales para lograr el éxito, la capacidad de percibir los sentimientos propios y los de los demás, y con ello tomar decisiones para regular la propia conducta, para actuar sin afectar a terceros. Es importante reconocer que la inteligencia emocional permite las habilidades para automotivarnos, las de perseverar, confiar en uno mismo, continuar en el camino cuando se presentan frustraciones, controlar nuestros impulsos, regular nuestros propios estados de ánimo para que éstos no controlen nuestras decisiones, evitar que la angustia interfiera con nuestras facultades racionales y la capacidad de empatizar y confiar en los demás. 
De lo anterior, se puede inferir que los hombres tienden con mayor frecuencia a internalizar las emociones y a mostrar una reacción fisiológica "emocional", es decir, presentar alguna reacción sintomática como temblar, sonrojarse, sudar, etcétera, pero sin mostrar una expresión emocional abierta.

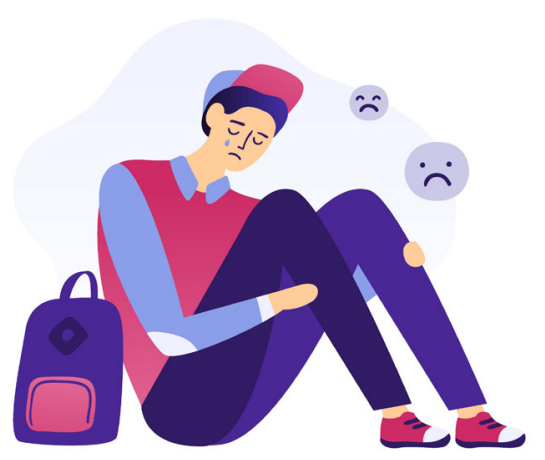

El control sobre las emociones en los hombres respalda una hegemonía de género y reafirma las estructuras de la sociedad patriarcal, donde son ellos quienes deben de mantener el control y en la cual las expresiones emocionales pueden interpretarse como debilidad. Por ello, prefieren inhibir su estado emocional para mantener el poder. Sin embargo, los hombres al internalizar sus emociones son susceptibles al dolor y heridas emocionales, mismas que podrían manifestarse en actos de violencia o aislamiento, quedando susceptibles a estados depresivos, e incluso al suicidio.

\section{Alcances y recomendaciones}

Las investigaciones sobre el tema apuntan a que existe una emocionalidad masculina inhibida o restrictiva, en la que la cultura determina cierta imposibilidad de expresión de algunas emociones. Es importante investigar qué consecuencias personales, sociales y culturales tiene esta emocionalidad restrictiva en los hombres. Asimismo, hay que abrir líneas de investigación desde el sector educativo para establecer pautas para una educación de la inteligencia emocional desde la infancia.

En el mismo sentido, y debido a que el control sobre las emociones en los hombres respalda una hegemonía de género, reafirma las estructuras de la sociedad patriarcal, favorece el aislamiento y entreteje dolor y miedo, es importante analizar en líneas de investigación futuras la relación existente entre la violencia de género con el control restrictivo de las emociones en los hombres. Esto para comprender si desempeña un papel clave en el aumento de la violencia de género y proponer soluciones.

Es necesario analizar las implicaciones de la inteligencia emocional en los hombres como sujetos de género, los estigmas socioculturales y su educación emocional desde la infancia en la familia, la cultura, las instituciones y la sociedad para romper el esquema patriarcal. 


\section{Referencias}

* Baena, G. (2003). Cómo desarrollar la inteligencia emocional infantil: guía para padres

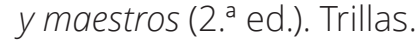

* Causa en común. (2021, 25 de enero). Los asesinatos se mantienen en niveles alarmantes, y los feminicidios, violencia familiar y narcomenudeo rompen récord: Causa en Común [boletín 2]. https://cutt.ly/vElylio

* Comisión de Ayuda al Refugiado en Euskadi (CEAR). (s/f). Patriarcado, Género y asilo. https://diccionario.cear-euskadi.org/patriarcado/

* Goleman, D. (1995). La inteligencia emocional. Porqué es más importante que el coeficiente intelectual. Le Libros. https://cutt.ly/PElucN2

* Grewal, D. y Salovey, P. (2006). Inteligencia emocional. Mente y cerebro, 16, 10-20. https://cutt.ly/GEluOZa

* Hernandez, J. M. (2020, 19 de mayo). La Voz de la IP. El Índice de Paz México 2020. Coparmex. https://cutt.ly/oEluKS3

* inegl. (2020, 8 de septiembre). Estadísticas a propósito del día mundial para la prevención del suicidio datos nacionales [Comunicado de prensa núm. 422/20]. https://cutt.ly/rEluOD|

* Instituto para la Economía y la Paz. (2021, mayo). Índice de Paz México 2021. Identificación y medición de los factores que impulsan la paz. https://www. visionofhumanity.org/resources/

* Ortega, N. A., Durán, K., Arrieta, M. G., Rivera, A. M. y R. García. (2013, octubre). Habilidades emocionales en hombres y mujeres estudiantes de ciencias de la salud. European Scientific Journal, 9(29), 326-341. https://cutt.ly/xElowol

* Ramírez,J.C.(2013). Masculinidadyemociones. Una aproximaciónasuconstrucción social. Acta Científica xxix Congreso de la Asociación Latinoamericana de Sociología 2013, Santiago, Chile. https://www.researchgate.net/publication/308796528

\section{Cómo CITAR ESTE ARTículo}

* Peralta Tellez, Liliana. (2021, noviembre-diciembre). La importancia de la inteligencia emocional en la población masculina. Revista Digital Universitaria (RDU), 22(6). http://doi.org/10.22201/cuaieed.16076079e.2021.22.6.3 